\title{
REGULARIZATION OF LINEAR LEAST SQUARES PROBLEMS BY TOTAL BOUNDED VARIATION
}

\author{
G. CHAVENT AND K. KUNISCH
}

ABstract. We consider the problem

(P) Minimize $\frac{1}{2}|T u-z|_{Y}^{2}+\frac{\alpha}{2}|u|_{L^{2}}^{2}+\beta \int_{\Omega}|\nabla u|$ over $u \in K \cap X$, where $\alpha \geq 0, \beta>0, K$ is a closed convex subset of $L^{2}(\Omega)$, and the last additive term denotes the $\mathrm{BV}$-seminorm of $u, T$ is a linear operator from $L^{2} \cap B V$ into the observation space $Y$. We formulate necessary optimality conditions for $(P)$. Then we show that $(P)$ admits, for given regularization parameters $\alpha$ and $\beta$, solutions which depend in a stable manner on the data $z$. Finally we study the asymptotic behavior when $\alpha=\beta \rightarrow 0$. The regularized solutions $\hat{u}_{\beta}$ of $(P)$ converge to the $L^{2} \cap$ $B V$ minimal norm solution of the unregularized problem. The rate of convergence is $\beta^{\frac{1}{2}}$ when the minimum-norm solution $\hat{u}$ is smooth enough.

\section{Problem Statement and FUnCtions of Bounded VARiation}

We consider the problem of finding approximate solutions to

$$
T u=z
$$

where $T$ is a bounded linear operator from $L^{2}(\Omega)$ to a Hilbert space $Y$ by using regularization techniques based on bounded variation functionals. Here $\Omega$ is a bounded domain in $\mathbb{R}^{n}$ with Lipschitzian boundary and $z \in Y$. The operator $T$ is not assumed to be injective and it may be compact. In this case approximate solutions that are stable with respect to $z$ can be obtained by solving the regularized problem.

$$
\min \frac{1}{2}|T u-z|_{Y}^{2}+\frac{\beta}{2}|u|_{L^{2}}^{2}
$$

or

$$
\min \frac{1}{2}|T u-z|_{Y}^{2}+\frac{\beta}{2}|\nabla u|_{L_{n}^{2}}^{2},
$$

for example. In (1.2) and (1.3) one refers to $\beta>0$ as the regularization parameter. Regularization by the square of a Hilbertian norm or seminorm as in (1.2) and (1.3) is well-studied. We refer to [2], [7], [8] and the references given there. While (1.2) and (1.3) have attractive analytical properties concerning the stability of their solution with respect to perturbations in the data $z$ and their asymptotic behavior as $\beta \rightarrow 0$ they can have serious practical disadvantages. The $L^{2}$-regularization term in (1.2) is not satisfactory

CEREMADE, Université Paris-Dauphine and INRIA, Rocquencourt.

Fachbereich Mathematik, Technische Universität Berlin and Institut für Mathematik, Universität Graz.

Received by the journal January 6, 1997. Accepted for publication October 23, 1997.

(C) Société de Mathématiques Appliquées et Industrielles. Typeset by AATEX. 
to dampen undesired oscillations in the solution $u$ which result from highly noisy data and/or compactness of $T$. Regularization with the square of the gradient as in (1.3) reduces these oscillations but it overregularizes the solution in the neighborhood of edges and corners. This is well-known in the context of image enhancement, see for example [11] and the references given there, and one can quickly convince oneself of this fact by a short MATLAB-code even with $T=$ identity. Due to these shortcomings with (1.2) and (1.3) regularization based on total bounded variation functionals has recently been investigated in several publications [3], [11], [13], for example. In this case the regularized problems are given by (variations of)

$$
\min \frac{1}{2}|T u-z|_{Y}^{2}+\beta \int_{\Omega}|\nabla u|
$$

where $\int_{\Omega}|\nabla u|$ denotes the bounded variation seminorm, which will be described at the end of this section. For the moment we may think of the term $\int_{\Omega}|\nabla u|$ as the $W^{1,1}(\Omega)$ seminorm. In the context of image enhancement (1.4) has proved to be extremely effective. Comparing (1.4) to (1.2) or (1.3) we immediately recognize that while (1.2) and (1.3) are quadratic, (1.4) is not. In fact, (1.4) is highly nonlinear and this results in interesting problems both analytically and numerically. In [3] basic facts concerning existence of solutions to (1.4), the optimality system, approximation of (1.4) by finite dimensional problems and algorithms that are specifically adapted for the nonlinear structure of problem (1.4) were presented. In the present analysis we focus on the problem of stability of the solutions to (1.4) with respect to perturbations in the data $z$ and on the asymptotic behavior as $\beta \rightarrow 0^{+}$.

Let us now specify the problem to be investigated:

$$
\min \frac{1}{2}|T u-z|_{Y}^{2}+\frac{\alpha}{2}|u|_{L^{2}}^{2}+\beta \int_{\Omega}|\nabla u| \text { over } u \in K \cap X,
$$

where $\alpha \geq 0, \beta>0, K$ is a closed, convex subset of $L^{2}(\Omega), X=L^{2}(\Omega) \cap$ $B V(\Omega)$, and $B V(\Omega)$ denotes the space of bounded variation functions to be defined below. The space $X$ endowed with the norm $|u|_{X}=|u|_{L^{2}}+|u|_{B V}$ is a Banach space. We shall see that due to the assumption that $\Omega$ is bounded $X$ and $B V(\Omega)$ are equivalent if $n \leq 2$. The use of the quadratic regularization term serves two purposes. First, for $\alpha>0$ it provides a coercive term for the subspace of constant functions which are in the kernel of the $\nabla$-operator (and can be in the kernel of $T$ ) and secondly it gives the possibility to distinguish the structure of stability results for quadratic regularization terms from that of the nonquadratic $B V$-term.

Let us now summarize results from the theory of functions of bounded variation that are relevant to our work. We refer to [3], [6], [12] for details. As mentioned above $\Omega$ is a bounded domain in $\mathbb{R}^{n}$ with Lipschitzian boundary. A function $u \in L^{1}(\Omega)$ is said to be of bounded variation if

$$
\sup \left\{\int_{\Omega} u(x) \operatorname{div} v(x) d x: v \in C_{0}^{\infty}(\Omega)^{n},|v(x)|_{\infty} \leq 1, x \in \Omega\right\}<\infty .
$$

Here $|\cdot|_{\infty}$ denotes the $l^{\infty}$-norm on $\mathbb{R}^{n}$. The $\sup$ in $(1.5)$ will be denoted by $\int_{\Omega}|\nabla u|$. The space of all functions in $L^{1}(\Omega)$ with bounded variation is EsAim: CoCV, December 1997, Vol. 2, PP. 359-376 
abbreviated by $B V(\Omega)$. Endowed with

$$
|u|_{B V}=|u|_{L^{1}}+\int_{\Omega}|\nabla u|
$$

$B V(\Omega)$ is a Banach space. Equivalently $B V(\Omega)$ can be introduced in terms of measures. Let $M(\Omega)$ denote the space of real Borel measures on $\Omega$ which is a Banach space when endowed with the norm

$$
|\mu|(\Omega)=\int_{\Omega}|\mu|, \mu \in M(\Omega),
$$

$|\mu|=\mu^{+}+\mu^{-}$being the total variation measure associated to $\mu$ and $|\mu|(\Omega)$ is the total variation of $\mu$ in $\Omega,[10]$. It is known that $M(\Omega)$ is the dual space of $C_{0}(\Omega)$, the space of continuous function vanishing on the boundary of $\Omega$ and

$$
\int_{\Omega}|\mu|=\sup \left\{\int_{\Omega} v(x) d \mu(x): v \in C_{0}(\Omega),|v|_{C(\Omega)} \leq 1\right\} .
$$

Thus $B V(\Omega)$ can equivalently be expressed as the space of functions $u \in$ $L^{1}(\Omega)$ for which $\partial_{x_{2}} u \in M(\Omega)$, for every $1 \leq i \leq n$, with the partial derivatives $\partial_{x_{i}}$ understood in the distributional sense. If the vector valued measure space $M^{n}(\Omega)$ is endowed with the $l^{1}$-norm, in the sense that

$$
\int_{\Omega}|\vec{\mu}|=\sum_{i=1}^{n} \int_{\Omega}\left|\vec{\mu}_{i}\right|, \vec{\mu} \in M^{n}(\Omega)
$$

then

$$
|u|_{B V}=|u|_{L^{1}}+\int_{\Omega}|\nabla u|=|u|_{L^{1}}+\sum_{i=1}^{n} \int_{\Omega}\left|\partial_{x_{i}} u\right|, \text { for } u \in B V(\Omega) .
$$

If instead of the $l^{\infty}$-norm on $\mathbb{R}^{n}$ in (1.5) one uses the $l^{p}$-norm, $1 \leq p<\infty$, then this induces an equivalent norm on $B V(\Omega)$.

Proposition 1.1.

a) If $\left\{u_{j}\right\}_{j=1}^{\infty} \subset B V(\Omega)$ and $\lim u_{j}=u$ in $L^{1}(\Omega)$ then

$$
\int_{\Omega}|\nabla u| \leq \liminf \int_{\Omega}\left|\nabla u_{j}\right| \text {. }
$$

b) For every $u \in B V(\Omega) \cap L^{r}(\Omega), r \in[1, \infty)$ there exists $\left\{u_{j}\right\}_{j=1}^{\infty} \subset C^{\infty}(\Omega)$ such that

$$
\lim u_{j}=u \text { in } L^{r}(\Omega) \text { and } \lim \int_{\Omega}\left|\partial_{x_{i}} u_{j}\right|=\int_{\Omega}\left|\partial_{x_{i}} u\right|, 1 \leq i \leq n .
$$

c) For every bounded sequence $\left\{u_{j}\right\}_{j=1}^{\infty} \subset B V(\Omega)$ there exists a subsequence $\left\{u_{j_{k}}\right\}_{k=1}^{\infty}$ and $u \in B V(\Omega)$ such that $\lim u_{j_{k}}=u$ in $L^{p}(\Omega), p \in$ $\left[1, \frac{n}{n-1}\right)$, if $n \geq 2$, and $\lim u_{j_{k}}=u$ in $L^{p}, p \in[1, \infty)$, if $n=1$. (Compactness).

d) There exists a constant $C=C(n)$ such that

$$
\left(\int_{\Omega}|u-\tilde{u}|^{\frac{n}{n-1}} d x\right)^{\frac{n-1}{n}} \leq C \int_{\Omega}|\nabla u|, \text { for all } u \in B V(\Omega),
$$

with $\tilde{u}=\frac{1}{|\Omega|} \int_{\Omega} u d x$. (Sobolev inequality). If $n=1$, then the $L^{n / n-1}$ norm is understood to be the $L^{\infty}$-norm. 
As a consequence of Proposition $1.1 \mathrm{~d}$ ) we have the following Corollary 1.2. If $n=1$ or 2 then $B V(\Omega)$ and $X$ are equivalent Banach spaces.

Sketch of proof of Proposition 1.1. The assertions of Proposition 1.1 are small modification of results given in [6]. The semicontinuity of the bounded variation functional asserted in a) is proved in ([6], Theorem 1.9). As for b) the proof of Theorem 1.17 in [6] can be generalized from $r=1$ to arbitrary $r \in[1, \infty)$. An application of Rellich's Theorem, see ([6], Theorem 1.19) implies c). Finally $\mathrm{d}$ ) is well-known for $u \in C^{\infty}(\Omega)$ [9]. For arbitrary $u \in B V(\Omega)$ there exists as a consequence of b) a sequence $u_{j} \in C^{\infty}(\Omega)$ such that $u_{j} \rightarrow u$ in $L^{1}(\Omega)$ and $\int_{\Omega}\left|\nabla u_{j}\right| \rightarrow \int_{\Omega}|\nabla u|$. Applying $\mathrm{d}$ ) to the sequence $\left\{u_{j}\right\}$ we obtain that $\left\{u_{j}\right\}_{j=1}^{\infty}$ is bounded in $L^{\frac{n}{n-1}}(\Omega)$ and hence $u_{j}$ converges weakly to $u$ in $L^{\frac{n}{n-1}}(\Omega)$ and $\lim \tilde{u}_{j}=\tilde{u}$. It follows that

$$
|u-\tilde{u}|_{L^{\frac{n}{n-1}}} \leq \underline{\lim }\left|u_{j}-\tilde{u}_{j}\right| \leq \lim C \int_{\Omega}\left|\nabla u_{j}\right|=C \int_{\Omega}|\nabla u| .
$$

The subsequent sections are organized as follows. In Section 2 sufficient conditions for existence of a unique solution to $(\mathrm{P})$ and the optimality system are given. Section 3 is dedicated to the stability of the solutions with respect to the data $z$. The asymptotic behavior of the solutions to $(\mathrm{P})$ as $\alpha \rightarrow 0, \beta \rightarrow 0$ is analyzed in Section 4 .

\section{EXISTENCE AND OPTIMALITY CONDITIONS}

We shall use the following hypothesis.

One of the following properties holds:

(i) $\alpha>0$,

(ii) $n=1$ or $n=2$, and const $\notin \operatorname{ker} T$,

(iii) $K$ is bounded in $L^{2}(\Omega)$.

THEOREM 2.1. If $(H)$ holds, then there exists a solution to $(P)$. If moreover $\alpha>0$ or $T$ is injective then the solution is unique.

Proof. Let $\left\{u_{j}\right\}_{j=1}^{\infty}$ be a minimizing sequence. If (H) (i) or (iii) hold, then $\left\{u_{j}\right\}$ is bounded in $L^{2}(\Omega)$. In the case of $(\mathrm{H})$ (ii) boundedness of $\left\{u_{j}\right\}$ in $L^{2}(\Omega)$ follows from Proposition $\left.1.1 \mathrm{~d}\right)$ and the fact that $\beta>0$. Thus in either case $(H)$ and the form of the cost functional imply that $\left\{u_{j}\right\}$ is bounded in $X$. By Proposition $1.1 \mathrm{c}$ ) there exists a subsequence of $\left\{u_{j}\right\}$ denoted by the same symbol and $\bar{u} \in X$, such that $\left\{u_{j}\right\}$ converges to $u$ strongly in $L^{1}(\Omega)$ and weakly in $L^{2}(\Omega)$. It follows that $\bar{u} \in K$. Moreover, $T$ maps weakly convergent sequence in $L^{2}(\Omega)$ into weakly convergent sequences in $Y$. By Proposition 1.1 a) we find

$$
\begin{aligned}
\frac{1}{2} \mid T & \bar{u}-\left.z\right|^{2}+\frac{\alpha}{2}|\bar{u}|^{2}+\beta \int_{\Omega}|\nabla \bar{u}| \\
& \leq \liminf \left\{\frac{1}{2}\left|T u_{j}-z\right|^{2}+\frac{\alpha}{2}\left|u_{j}\right|_{L^{2}}^{2}+\beta \int_{\Omega}\left|\nabla u_{j}\right|\right\} \\
& \leq \inf \left\{\frac{1}{2}|T u-z|^{2}+\frac{\alpha}{2}|u|_{L^{2}}^{2}+\beta \int_{\Omega}|\nabla u|: u \in K\right\}
\end{aligned}
$$


and thus $\bar{u}$ is a solution to $(\mathrm{P})$. Concerning uniqueness we note that in the case $\alpha>0$ the cost functional of $(\mathrm{P})$ is strictly convex and hence $\bar{u}$ is unique. In the case of injectivity of $T$ the cost functional of $(P)$ is again strictly convex and uniqueness of $\bar{u}$ follows.

We now specify the optimality system for $(\mathrm{P})$.

Theorem 2.2. Let $\bar{u} \in K \cap X$. Then $\bar{u}$ is a solution of $(P)$ if and only if there exists $\bar{\lambda}$ in the dual space of $M^{n}(\Omega)$ such that

$$
\begin{array}{r}
\left(T^{*}(T \bar{u}-z)+\alpha \bar{u}, u-\bar{u}\right)_{L^{2}(\Omega)}-\beta\langle\operatorname{div} \bar{\lambda}, u-\bar{u}\rangle_{B V^{*}, B V} \geq 0 \\
\text { for all } u \in K \cap X \\
\langle\bar{\lambda}, \mu-\nabla \bar{u}\rangle_{M^{n, *}, M^{n}}+\int_{\Omega}|\nabla \bar{u}| \leq \int_{\Omega}|\mu|, \\
\text { for all } \mu \in M^{n}(\Omega) .
\end{array}
$$

Here $T^{*}$ denotes the adjoint of $T \in \mathcal{L}\left(L^{2}(\Omega), Y\right)$ and - div stands for the conjugate of $\nabla: B V(\Omega) \rightarrow M^{n}(\Omega)$, i.e.

$$
\langle-\operatorname{div} \bar{\lambda}, u\rangle_{B V^{*}, B V}=\langle\bar{\lambda}, \nabla u\rangle_{M^{n, *}, M^{n}} \text { for } u \in B V(\Omega) \text {. }
$$

The proof can be obtained with only minor modifications from that of ([3], Theorem 2.2) and it is therefore omitted. Let us note that (2.2) is equivalent to

$$
\langle\bar{\lambda}, \nabla \bar{u}\rangle=\int_{\Omega}|\nabla \bar{u}|
$$

and

$$
\langle\bar{\lambda}, \mu\rangle \leq \int_{\Omega}|\mu| \text { for all } \mu \in M^{n}(\Omega) .
$$

Alternatively (2.2) can be expressed as $\bar{\lambda} \in \partial \varphi(\nabla \bar{u})$ where $\partial \varphi$ is the subdifferential of $\varphi: M^{n}(\Omega) \rightarrow \mathbb{R}$ given by

$$
\varphi(\mu)=\sum_{i=1}^{n} \int_{\Omega}\left|\mu_{i}\right| .
$$

Note that (2.4) implies that the restriction to $L_{n}^{1}$ of $\mu \rightarrow\langle\bar{\lambda}, \mu\rangle$ can be identified with an element $\bar{\lambda}$ in $L_{n}^{\infty}(\Omega)$ with $|\bar{\lambda}|_{L_{n}^{\infty}} \leq 1$.

The Lagrange multiplier $\bar{\lambda}$ of Theorem 2.2 is not unique. In the following theorem we give a necessary and sufficient optimality system that involves a unique Lagrange multiplier $\bar{q} \in H^{1}(\Omega)$. Let us recall some function spaces involving the divergence operator $[5]$ :

$$
H(\operatorname{div})=\left\{v \in L_{n}^{2}(\Omega): \operatorname{div} v \in L^{2}(\Omega)\right\},
$$

which is a Hilbert space for the norm

$$
|v|_{H(\operatorname{div})}=\left(|v|_{L_{n}^{2}}^{2}+|\operatorname{div} v|_{L^{2}}^{2}\right)^{1 / 2}
$$

and the closed subspace of $H($ div $)$ :

$$
H_{0}(\operatorname{div})=\overline{\mathcal{D}(\Omega)^{n}}
$$

where $\mathcal{D}(\Omega)^{n}$ denotes the space of infinitely differentiable vector-valued functions with support in $\Omega$ and the closure is taken in the norm of $H(\mathrm{div})$. The trace operator $\tau(v)=v \cdot n \mid \Gamma$ can be extended as continuous linear operator from $H$ (div) to $H^{-1 / 2}(\Gamma)([5], \S 2)$. Here $n$ denotes the outer unit normal to 
the Lipschitzian boundary $\Gamma$ of $\Omega$. The space $H_{0}$ (div) can be characterized as

$$
H_{0}(\operatorname{div})=\{v \in H(\operatorname{div}): \tau(v)=0\} .
$$

Theorem 2.3. Let $K=X$ and let $\bar{u}$ be a solution to $(P)$. Then there exists a unique $\bar{q} \in H^{1}(\Omega)$ with $\int_{\Omega} \bar{q} d x=0$ such that for $\tilde{\lambda}=\nabla \bar{q}$ we have $\tilde{\lambda} \in$ $H_{0}($ div $),|\tilde{\lambda}|_{L_{n}^{2}} \leq \sqrt{\text { meas }(\Omega)}$

$$
\begin{gathered}
\left(T^{*} T+\alpha\right) \bar{u}-T^{*} z=\beta \operatorname{div} \tilde{\lambda} \text { in } L^{2}(\Omega) \\
(-\operatorname{div} \tilde{\lambda}, u-\bar{u})_{L^{2}(\Omega)}+\int_{\Omega}|\nabla \bar{u}| \leq \int_{\Omega}|\nabla u| \text { for all } u \in X .
\end{gathered}
$$

Conversely, if there exists a pair $(\bar{u}, \tilde{\lambda}) \in X \times H_{0}$ (div) such that (2.5), (2.6) hold, then $\bar{u}$ is a solution to $(P)$.

REMARK 2.4. From the proof it will follow that $\tilde{\lambda}$ of Theorem 2.3 is obtained from the Lagrange multiplier $\bar{\lambda}$ of Theorem 2.2 by restriction of the functional $\mu \rightarrow\langle\bar{\lambda}, \mu\rangle_{M^{n, *}, M^{n}}$ to $L_{n}^{2}(\Omega)$ and subsequent projection of $\bar{\lambda}$ onto the orthogonal complement in $L_{n}^{2}(\Omega)$ of the divergence-free space. We recall the decomposition of $L_{n}^{2}(\Omega)$ as

$$
L_{n}^{2}(\Omega)=H \oplus H^{\perp},
$$

where $H=\left\{v \in H_{0}(\operatorname{div}): \operatorname{div} v=0\right\}$, and $H^{\perp}$ denotes the orthogonal complement of $H$ in $L_{n}^{2}(\Omega)$. Accordingly the restriction to $L_{n}^{2}(\Omega)$ of $\bar{\lambda}$ of Theorem 2.2 can be decomposed as $\bar{\lambda}_{1}+\bar{\lambda}_{2} \in H \oplus H^{\perp}$ and $\bar{\lambda}_{2}$ can be identified with the Lagrange multiplier $\tilde{\lambda}$ of Theorem 2.3.

Proof of Theorem 2.3. By Theorem 2.2 there exists $\bar{\lambda} \in M^{n}(\Omega)^{*}$, the dual of $M^{n}(\Omega)$, such that (2.1) and (2.2) hold. Since $K=X$ we find

$$
\left(\left(T^{*} T+\alpha\right) \bar{u}-T^{*} z, v\right)_{L^{2}}=-\beta\langle\bar{\lambda}, \nabla v\rangle_{M^{n, *}, M^{n}} \text { for all } v \in X .
$$

Let us consider the restriction of the functional $\mu \rightarrow\langle\bar{\lambda}, \mu\rangle_{M^{n, *}, M^{n}}$ to $L_{n}^{2}(\Omega)$. By the Riesz representation theorem it can be identified with an element of $L_{n}^{2}(\Omega)$ which we denote by $\hat{\lambda}$. Due to $(2.4)$ we find that $|\hat{\lambda}|_{L_{n}^{2}} \leq \sqrt{\text { meas }(\Omega)}$. Taking the distributional derivative in

$$
\left(\left(T^{*} T+\alpha\right) \bar{u}-T^{*} z, v\right)_{L^{2}}=-\beta(\hat{\lambda}, \nabla v)_{L_{n}^{2}} \text { for all } v \in H^{1}(\Omega),
$$

it follows that div $\hat{\lambda}$ can be considered as bounded linear functional on $L^{2}(\Omega)$. Hence $\hat{\lambda}$ can be identified with an element of $H(\mathrm{div})$ and

$$
\left(T^{*} T+\alpha\right) \bar{u}-T^{*} z=\beta \operatorname{div} \hat{\lambda} \text { in } L^{2}(\Omega) .
$$

Combining (2.7) and (2.8) it follows that

$$
(\operatorname{div} \hat{\lambda}, v)_{L^{2}}=-\langle\bar{\lambda}, \nabla v\rangle_{M^{n, *}, M^{n}} \text { for all } v \in X
$$

Let us argue that $\hat{\lambda} \in H_{0}$ (div). By Green's formula and (2.9) we have

$$
(\operatorname{div} \hat{\lambda}, v)_{L^{2}}+(\hat{\lambda}, \nabla v)_{L^{2}}=\int_{\Gamma} v \hat{\lambda} n d s=0 \text { for every } v \in \mathcal{D}(\bar{\Omega}) .
$$

Esaim: Cocv, December 1997, Vol. 2, PP. 359-376 
Hence the trace of $\hat{\lambda}$ satisfies $\tau(\hat{\lambda})=0$ and $\hat{\lambda} \in H_{0}$ (div). Let $H=\{v \in$ $\left.H_{0}(\operatorname{div}): \operatorname{div} v=0\right\}$. Since $H$ is a closed subspace of $L_{n}^{2}(\Omega)$, we have

$$
L_{n}^{2}=H \oplus H^{\perp},
$$

with $H^{\perp}$ the orthogonal complement of $H$ in $L_{n}^{2}(\Omega)$. It is well-known that $H^{\perp}=\left\{\nabla q: q \in H^{1}(\Omega)\right\}[5]$. Let us decompose

$$
\hat{\lambda}=\lambda_{1}+\lambda_{2} \in H \oplus H^{\perp},
$$

with $\operatorname{div} \lambda_{1}=0$ and $\lambda_{2}=\nabla \bar{q}$. The element $\bar{q}$ is unique when normalized by $\int_{\Omega} \bar{q} d x=0$. Then (2.5) follows from (2.8). To verify (2.6) let us observe that by $(2.2)$

$$
\langle\bar{\lambda}, \nabla(u-\bar{u})\rangle_{M^{n, *}, M^{n}}+\int_{\Omega}|\nabla \bar{u}| \leq \int_{\Omega}|\nabla u| \text { for all } u \in X
$$

and by (2.9)

$$
\left(-\operatorname{div} \lambda_{2}, u-\bar{u}\right)_{L^{2}}+\int_{\Omega}|\nabla \bar{u}| \leq \int_{\Omega}|\nabla u| \text { for all } u \in X .
$$

Finally concerning the $L_{n}^{2}$-norm of $\lambda_{2}$ we have due to the orthogonal decomposition (2.10)

$$
\left|\lambda_{2}\right|_{L_{n}^{2}} \leq|\bar{\lambda}|_{L_{n}^{2}} \leq \sqrt{\operatorname{meas}(\Omega)}
$$

This concludes the first part of the proof with $\tilde{\lambda}=\lambda_{2}$.

Conversely assume that $(\bar{u}, \tilde{\lambda}) \in X \times H_{0}$ (div) satisfies (2.5), (2.6), and let $u$ be an arbitrary element in $X$. Let $F$ denote the cost functional in $(\mathrm{P})$. Then by (2.5), (2.6)

$$
\begin{aligned}
& F(u)-F(\bar{u}) \geq\left(\left(T^{*} T+\alpha\right) \bar{u}, u-\bar{u}\right)_{L^{2}}+\beta \int_{\Omega}|\nabla u|-\beta \int_{\Omega}|\nabla \bar{u}| \\
& \quad=\beta(\operatorname{div} \tilde{\lambda}, u-\bar{u})-\beta(\operatorname{div} \tilde{\lambda}, u-\bar{u}) \geq 0
\end{aligned}
$$

and $\bar{u}$ is a solution to $(\mathrm{P})$.

Corollary 2.5. Under the assumptions of Theorem 2.3, $\bar{q}$ is the unique variational solution satisfying $\int_{\Omega} \bar{q} d x=0$ to the Neumann problem

$$
\left\{\begin{aligned}
-\beta \Delta q & =\left(T^{*} T+\alpha\right) \bar{u}+T^{*} z \text { in } \Omega \\
\frac{\partial q}{\partial n} & =0 \text { on } \Gamma .
\end{aligned}\right.
$$

The compatibility condition is clearly satisfied since

$$
\int_{\Omega}\left(\left(T^{*} T+\alpha\right) \bar{u}-T^{*} z\right) d x=0
$$

by (2.7) with $v=1$. If the boundary of $\Omega$ is $C^{1,1}$-smooth then $\bar{q} \in H^{2}(\Omega)$.

Corollary 2.6. Under the assumption of Theorem 2.3

$$
(-\operatorname{div} \tilde{\lambda}, \bar{u})_{L^{2}(\Omega)}=\int_{\Omega}|\nabla \bar{u}|
$$

and

$$
(\operatorname{div} \tilde{\lambda}, u)_{L^{2}(\Omega)} \leq \int_{\Omega}|\nabla u| \text { for all } u \in X .
$$

Equality (2.12) follows from (2.6) with $u=0$ and $u=2 \bar{u}$. By (2.6) and (2.12) one obtains (2.13). 


\section{Stability With ResPeCt TO DATA}

This section is dedicated to the analysis of the stability of the solution to $(\mathrm{P})$ with respect to the data $z$. It will be convenient to denote $(\mathrm{P})$ by $\left(P_{z}\right)$ and solutions to $\left(P_{z}\right)$ by $u_{z}$. Let us first give the stability result that can be attained from a-priori estimates.

TheOrEm 3.1. Assume that (H) holds, let $\left\{z_{n}\right\}$ be a sequence of observations in $Y$ converging to $z$, and let $\left\{u_{z_{n}}\right\}$ denote a sequence of solutions to $\left(P_{z_{n}}\right)$. Then there exists a subsequence $\left\{u_{z_{n_{k}}}\right\}$ of $\left\{u_{z_{n}}\right\}$ and $u_{z} \in L^{2}(\Omega)$ such that

$$
\lim u_{z_{n_{k}}}=u_{z} \text { weakly in } L^{2}(\Omega) \text { and } \lim \int_{\Omega}\left|\nabla u_{z_{n_{k}}}\right|=\int_{\Omega}\left|\nabla u_{z}\right|,
$$

and every such sequence has the property that $u_{z}$ is a solution to $(P)$. If (H) (i) holds then $\lim u_{z_{n_{k}}}=u_{z}$ strongly in $L^{2}(\Omega)$.

Proof. For simplicity let us denote $u_{n}=u_{z_{n}}$. We have for every $u \in K$

$$
\begin{array}{r}
\frac{1}{2}\left|T u_{n}-z_{n}\right|_{Y}^{2}+\frac{\alpha}{2}\left|u_{n}\right|_{L^{2}}^{2}+\beta \int_{\Omega}\left|\nabla u_{n}\right| \leq \frac{1}{2}\left|T u-z_{n}\right|_{Y}^{2} \\
+\frac{\alpha}{2}|u|_{L^{2}}^{2}+\beta \int_{\Omega}|\nabla u| .
\end{array}
$$

If (H) (i) or (iii) hold then it follows directly that $\left\{u_{n}\right\}$ is bounded in $X$. In the case of $(\mathrm{H})$ (ii) boundedness of $\left\{u_{n}\right\}$ in $X$ follows from Proposition $1.1 \mathrm{~d}$ ). Thus there exists a subsequence of $\left\{u_{n}\right\}$ denoted, for simplicity by the same symbol and $u_{z} \in L^{2}(\Omega)$ such that

$$
\lim u_{n}=u_{z} \text { weakly in } L^{2}(\Omega) \text { and } \lim u_{n}=u_{z} \text { in } L^{1}(\Omega) .
$$

It follows that $u_{z} \in K$ and

$$
\lim T u_{n}=T u_{z} \text { weakly in } Y \text { and } \int_{\Omega}\left|\nabla u_{z}\right| \leq \underline{\lim } \int_{\Omega}\left|\nabla u_{n}\right| .
$$

We can now pass to the limit in (3.2) to obtain

$$
\frac{1}{2}\left|T u_{z}-z\right|_{Y}^{2}+\frac{\alpha}{2}\left|u_{z}\right|_{L^{2}}^{2}+\int_{\Omega}\left|\nabla u_{z}\right| \leq \frac{1}{2}|T u-z|_{Y}^{2}+\frac{\alpha}{2}|u|_{L^{2}}^{2}+\beta \int|\nabla u|
$$

for every $u \in K$ and hence $u_{z}$ is a solution to $\left(P_{z}\right)$. Let us show next the second convergence statement in (3.1). We have

$$
\begin{aligned}
& \frac{1}{2}\left|T u_{z}-z\right|^{2}+\frac{\alpha}{2}\left|u_{z}\right|^{2}+\varlimsup i m \beta \int_{\Omega}\left|\nabla u_{n}\right| \\
& \leq \underline{\lim } \frac{1}{2}\left|T u_{n}-z_{n}\right|^{2}+\underline{\lim } \frac{\alpha}{2}\left|u_{n}\right|^{2}+\bar{\varlimsup} \beta \int_{\Omega}\left|\nabla u_{n}\right| \\
& \leq \mp\left(\frac{1}{2}\left|T u_{n}-z_{n}\right|^{2}+\frac{\alpha}{2}\left|u_{n}\right|^{2}+\beta \int_{\Omega}\left|\nabla u_{n}\right|\right) \\
& \leq \varlimsup\left(\frac{1}{2}\left|T u_{z}-z_{n}\right|^{2}+\frac{\alpha}{2}\left|u_{z}\right|^{2}+\beta \int_{\Omega}\left|\nabla u_{z}\right|\right) \\
& =\frac{1}{2}\left|T u_{z}-z\right|^{2}+\frac{\alpha}{2}\left|u_{z}\right|^{2}+\beta \int_{\Omega}\left|\nabla u_{z}\right|,
\end{aligned}
$$


and hence $\varlimsup \int_{\Omega}\left|\nabla u_{n}\right| \leq \int_{\Omega}\left|\nabla u_{z}\right|$, which together with $\int_{\Omega}\left|\nabla u_{z}\right|$ $\leq \underline{\lim } \int_{\Omega}\left|\nabla u_{n}\right|$ implies the second statement in (3.1). If (H) (i) holds then one shows similarly that $\lim \left|u_{n}\right|_{L^{2}}=\left|u_{z}\right|$, which together with weak convergence implies strong convergence in $L^{2}(\Omega)$ of $u_{n}$ to $u_{z}$.

The following lemma will be useful.

LemMa 3.2. For every pair of solutions $u_{z}$ and $u_{\bar{z}}$ with associated Lagrange multipliers $\lambda_{z}$ and $\lambda_{\bar{z}}$ we have

$$
\left\langle\lambda_{z}-\lambda_{\bar{z}}, \nabla\left(u_{z}-u_{\bar{z}}\right)\right\rangle_{M^{n, *}, M^{n}} \geq 0 .
$$

Proof. Since $\lambda_{z} \in \partial \varphi\left(\nabla u_{z}\right)$ and $\lambda_{\bar{z}} \in \partial \varphi\left(\nabla u_{\bar{z}}\right)$ the Lemma follows from a wellknown property of subdifferentials.

Theorem 3.3. Let (H) (i) hold. Then for every pair $z, \bar{z} \in Y$

$$
\left|u_{z}-u_{\bar{z}}\right|_{L^{2}} \leq \frac{1}{\alpha}\|T\|_{\mathcal{L}\left(L^{2}, Y\right)}|z-\bar{z}|_{Y} .
$$

Proof. From (2.1) of Theorem 2.2 we have

$$
\begin{aligned}
& \left\langle\left(T^{*} T+\alpha\right) u_{z}-T^{*} z-\beta \operatorname{div} \lambda_{z}, u_{\bar{z}}-u_{z}\right\rangle \geq 0 \\
& \left\langle\left(T^{*} T+\alpha\right) u_{\bar{z}}-T^{*} \bar{z}-\beta \operatorname{div} \lambda_{\bar{z}}, u_{z}-u_{\bar{z}}\right\rangle \geq 0 .
\end{aligned}
$$

The duality pairing $\langle\cdot, \cdot\rangle$ must be interpreted as in (2.1) and hence

$$
\left\langle\left(T^{*} T+\alpha\right)\left(u_{z}-u_{\bar{z}}\right)-T^{*}(z-\bar{z})-\beta \operatorname{div}\left(\lambda_{z}-\lambda_{\bar{z}}\right), u_{\bar{z}}-u_{z}\right\rangle \geq 0 .
$$

Lemma 3.2 implies that

$$
\left\langle\left(T^{*} T+\alpha\right)\left(u_{z}-u_{\bar{z}}\right)-T^{*}(z-\bar{z}), u_{z}-u_{\bar{z}}\right\rangle \leq \beta\left\langle\lambda_{z}-\lambda_{\bar{z}}, \nabla\left(u_{\bar{z}}-u_{z}\right)\right\rangle \leq 0,
$$

and hence

$$
\alpha\left|u_{z}-u_{\bar{z}}\right|^{2} \leq \| T^{*}||\left|u_{z}-u_{z}\right||\bar{z}-z|
$$

and the result follows.

Let $B_{r}$ denote the ball with center at the origin and radius $r$ in $Y$. Theorem 3.4. Let $(H)$ (i) hold and let $r>0$. Then

$$
\beta\left|\int_{\Omega}\right| \nabla u_{z}\left|-\int_{\Omega}\right| \nabla u_{\bar{z}}|| \leq \frac{r}{\alpha}\left(2+\frac{1}{\alpha}\right) \max \left(1,\|T\|^{4}\right)|z-\bar{z}|,
$$

for every $z, \bar{z} \in B_{r}$.

Proof. From Theorem 3.3 we conclude that

$$
\left|u_{z}\right|_{L^{2}} \leq \frac{r}{\alpha}\|T\| \text { for every } z \in B_{r} .
$$

Utilizing the fact that $u_{z}$ is a solution to $\left(P_{z}\right)$ we find

$$
\beta\left(\int_{\Omega}\left|\nabla u_{z}\right|-\int_{\Omega}\left|\nabla u_{\bar{z}}\right|\right) \leq \frac{1}{2}\left[\left|T u_{\bar{z}}-z\right|^{2}-\left|T u_{z}-z\right|^{2}+\alpha\left|u_{\bar{z}}\right|^{2}-\alpha\left|u_{z}\right|^{2}\right] .
$$

Similarly, since $u_{\bar{z}}$ is a solution to $\left(P_{\bar{z}}\right)$

$$
\beta\left(\int_{\Omega}\left|\nabla u_{\bar{z}}\right|-\int_{\Omega}\left|\nabla u_{z}\right|\right) \leq \frac{1}{2}\left[\left|T u_{z}-\bar{z}\right|^{2}-\left|T u_{\bar{z}}-\bar{z}\right|^{2}+\alpha\left|u_{z}\right|^{2}-\alpha\left|u_{\bar{z}}\right|^{2}\right] .
$$


Combining these two inequalities implies by Theorem 3.3 and (3.4) that

$$
\begin{aligned}
\beta\left|\int_{\Omega}\right| \nabla u_{z}\left|-\int_{\Omega}\right| \nabla u_{\bar{z}}|| \leq & \frac{\alpha}{2}\left|u_{z}-u_{\bar{z}}\right|\left|u_{z}+u_{\bar{z}}\right| \\
& \quad+\frac{1}{2}\left|T u_{z}-T u_{\bar{z}}\right|\left(\left|T u_{z}+T u_{\bar{z}}\right|+2 r\right) \\
\leq & \frac{r}{\alpha}\|T\|^{2}|z-\bar{z}|+\|T\|^{3}\left|u_{z}-u_{\bar{z}}\right|\left(\frac{r}{\alpha}+r\right) \\
= & \frac{r}{\alpha}\left(2+\frac{1}{\alpha}\right)\|T\|^{4}|z-\bar{z}|,
\end{aligned}
$$

and the desired estimate follows.

The last aspect that we address in this section is the stability of the dual variable $\lambda=\lambda_{z}$ with respect to perturbations in the data $z$. It will be assumed that $(\mathrm{H})$ (i) is satisfied. This implies existence of a unique solution $u_{z}$ to $\left(P_{z}\right)$ for every $z \in Y$.

Theorem 3.5. Assume that $(H)$ (i) holds and that $K=X$. Let $\left\{z_{n}\right\}$ be a sequence in $Y$ with limit $z$, and let $\left(u_{z_{n}}, \lambda_{z_{n}}\right)$ be the solutions and Lagrange multipliers to $\left(P_{z_{n}}\right)$ according to Theorem 2.2. Then $u_{z_{n}}$ converges strongly in $L^{2}(\Omega)$ to the solution $u_{z}$ of $\left(P_{z}\right)$ and $\lim \int_{\Omega}\left|\nabla u_{z_{n}}\right|=\int_{\Omega}\left|\nabla u_{z}\right|$. Furthermore there exists a subsequence $\left\{\lambda_{z_{n_{k}}}\right\}$ of $\left\{\lambda_{z_{n}}\right\}$ converging weakly in $H$ (div) and weak $k^{*}$ in $L^{\infty}(\Omega)$ to some $\lambda_{z} \in L_{n}^{\infty}(\Omega) \cap H_{0}$ (div) and for every such cluster point we have

$$
\begin{gathered}
\left(T^{*} T+\alpha\right) u_{z}=T^{*} z+\beta \operatorname{div} \lambda_{z} \text { in } L^{2}(\Omega), \\
-\left(\operatorname{div} \lambda_{z}, u_{z}\right)_{L^{2}}=\int_{\Omega}\left|\nabla u_{z}\right|, \text { and } \\
\left(\lambda_{z}, \mu\right)_{L^{\infty}, L^{1}} \leq \int_{\Omega}|\mu| d x, \text { for all } \mu \in L_{n}^{1}(\Omega) .
\end{gathered}
$$

Proof. The first part of the claim follows from Theorem 3.1. For simplicity we shall write $\left(u_{n}, \lambda_{n}\right)$ in place of $\left(u_{z_{n}}, \lambda_{z_{n}}\right)$. The optimality systems for $\left(P_{z_{n}}\right)$ are given by

$$
\begin{gathered}
\left(T^{*} T+\alpha\right) u_{n}=T^{*} z+\beta \operatorname{div} \lambda_{n}, \\
\left\langle\lambda_{n}, \nabla u_{n}\right\rangle_{M^{n, *}, M^{n}}=\int_{\Omega}\left|\nabla u_{n}\right|, \\
\left\langle\lambda_{n}, \mu\right\rangle_{M^{n, *}, M^{n}} \leq \int_{\Omega}|\mu|, \text { for all } \mu \in M^{n}(\Omega) .
\end{gathered}
$$

Since $L_{n}^{1}(\Omega) \subset M^{n}(\Omega)$, the restrictions of $\mu \rightarrow\left\langle\lambda_{n}, \mu\right\rangle_{M^{n, *}, M^{n}}$ to $L_{n}^{1}(\Omega)$ can be identified with elements in $L_{n}^{\infty}(\Omega)$ with the property that $\left|\lambda_{n}\right|_{L_{n}^{\infty}} \leq 1$ for all $n$, by (3.10). From (3.8) it follows that $\left\{\lambda_{n}\right\}$ is bounded in $H_{0}$ (div) and thus there exists a subsequence of $\left\{\lambda_{n}\right\}$ denoted by the same symbol and $\lambda_{z} \in L_{n}^{\infty}(\Omega) \cap H_{0}$ (div) such that $\left|\lambda_{z}\right|_{L_{n}^{\infty}} \leq 1, \lambda_{n} \rightarrow \lambda_{z}$ weak $^{*}$ in $L_{n}^{\infty}(\Omega)$ and $\lambda_{n} \rightarrow \lambda_{z}$ weakly in $H_{0}$ (div). Taking the limit in (3.8) implies (3.5) and Esaim: Cocv, December 1997, Vol. 2, PP. 359-376 
(3.7) is equivalent to $\left|\lambda_{z}\right|_{L_{n}^{\infty}} \leq 1$. To verify (3.6) one argues as in the proof of Theorem 2.3 - see (2.9) — that (3.9) implies

$$
-\left(\operatorname{div} \lambda_{n}, u_{n}\right)_{L^{2}}=\int\left|\nabla u_{n}\right| \text {. }
$$

Taking the limit in (3.11) one obtains (3.6).

It is simple to pass to the limit in the optimality system $(2.5),(2.6)$ and to obtain the following result.

Theorem 3.6. Let the assumptions of Theorem 3.5 hold and let $\left(u_{z_{n}}, \tilde{\lambda}_{n}\right)$ denote the solutions to $\left(P_{z_{n}}\right)$ with the associated Lagrange multipliers according to Theorem 2.3. Then $\tilde{\lambda}_{z_{n}}$ converges strongly in $L_{n}^{2}(\Omega)$ to $\tilde{\lambda}_{z}=\nabla q_{z}$ and weakly in $H_{0}$ (div) and

$$
\begin{aligned}
& \left(T^{*} T+\alpha\right) u_{z}=T^{*} z+\beta \operatorname{div} \tilde{\lambda}_{z} \text { in } L^{2}(\Omega) \\
& \left(-\operatorname{div} \tilde{\lambda}_{z}, u-u_{z}\right)_{L^{2}}+\int_{\Omega}\left|\nabla u_{z}\right| \leq \int_{\Omega}|\nabla u| \text { for all } u \in X .
\end{aligned}
$$

\section{ASYMPTOTIC ANALYSIS}

In this section convergence and rate of convergence of the solutions of the regularized problems as the regularization parameter tends to zero is analyzed. We consider the case where $\alpha=\beta$ and for convenience we repeat the problem formulation:

$$
\min \frac{1}{2}|T u-z|_{Y}^{2}+\frac{\beta}{2}|u|_{L^{2}}^{2}+\beta \int_{\Omega}|\nabla u| \text { over } u \in K \cap X .
$$

From Theorem 2.1 it is known that $\left(\mathrm{P}_{\beta}\right)$ admits a unique solution $u_{\beta}$ for every $\beta>0$ and $z \in Y$. The unregularized least squares problem is given by

$$
\min \frac{1}{2}|T u-z|_{Y}^{2} \text { over } u \in K \cap X \text {. }
$$

Let us henceforth assume that $z \in Y$ is fixed and that $z$ has a projection $\hat{z} \in Y$ on range $T(K \cap X)$. Since $K$ is assumed to be convex this projection is necessarily unique. It then follows that $\left(\mathrm{P}_{0}\right)$ admits a solution and we define the set of solutions

$$
\mathcal{S}=\{u \in K \cap X: T u=\hat{z}\} .
$$

Let us introduce the notion of minimal solution to $\left(\mathrm{P}_{0}\right)$ in $\mathcal{S}$. For that purpose we consider

$$
\min \frac{1}{2}|u|_{L^{2}}^{2}+\int_{\Omega}|\nabla u|, \quad u \in \mathcal{S} .
$$

By Proposition 1.1 it is simple to argue the existence of a solution to (Q). Due to the strict convexity of the $L^{2}$-part of the cost functional in (Q) this 
solution is unique. It will be denoted by $\hat{u}_{z}$ and it is called the minimal solution of $\left(\mathrm{P}_{0}\right)$.

Proposition 4.1. There exists $\Lambda_{z}$ in the dual of $M^{n}(\Omega)$ such that

$$
\begin{array}{llll}
\left(\hat{u}_{z}, u-\hat{u}_{z}\right)_{L^{2}}-\left\langle\operatorname{div} \Lambda_{z}, u-\hat{u}_{z}\right\rangle_{B V^{*}, B V} \geq 0 & \text { for all } & u \in \mathcal{S}, \\
\left\langle\Lambda_{z}, \mu-\nabla \hat{u}_{z}\right\rangle_{M^{n, *}, M^{n}}+\int_{\Omega}\left|\nabla \hat{u}_{z}\right| \leq \int_{\Omega}|\mu| & \text { for all } & \mu \in M^{n}(\Omega) .
\end{array}
$$

Here, as in Section 2, -div is the adjoint to $\nabla: B V(\Omega) \rightarrow M^{n}(\Omega)$.

Let us give a first result on the behavior of the solutions $u_{\beta}$ as a function of $\beta$.

Proposition 4.2.

(i) $\frac{1}{2}\left|u_{\beta}\right|_{L^{2}}^{2}+\int_{\Omega}\left|\nabla u_{\beta}\right| \leq \frac{1}{2}\left|\hat{u}_{z}\right|_{L^{2}}^{2}+\int_{\Omega}\left|\nabla \hat{u}_{z}\right|$, for every $\beta>0$,

(ii) $\left|T \hat{u}_{z}-z\right|_{Y} \leq\left|T u_{\beta}-z\right|_{Y}$, for every $\beta>0$,

(iii) $\lim _{\beta \rightarrow 0^{+}} u_{\beta}=\hat{u}_{z}$ strongly in $L^{p}(\Omega), p<\frac{n}{n-2}, \lim _{\beta \rightarrow 0^{+}} u_{\beta}=\hat{u}_{z}$ weakly in $L^{2}(\Omega)$ and $\lim _{\beta \rightarrow 0^{+}} T u_{\beta}=T \hat{u}_{z}$ strongly in $Y$.

(iv) If $z \in$ range $(T)$, then $\left|T u_{\beta}-z\right|=o(\sqrt{\beta})$.

Proof. The second assertion follows from the fact that $\hat{u}_{z} \in \mathcal{S}$. To verify (i) we use (ii) and the fact that

$$
\begin{aligned}
& \frac{1}{2}\left|T u_{\beta}-z\right|^{2}+\frac{\beta}{2}\left|u_{\beta}\right|_{L^{2}}^{2}+\beta \int_{\Omega}\left|\nabla u_{\beta}\right| \\
& \quad \leq \frac{1}{2}\left|T \hat{u}_{z}-z\right|_{Y}^{2}+\frac{\beta}{2}\left|\hat{u}_{z}\right|_{L^{2}}^{2}+\beta \int_{\Omega}\left|\nabla \hat{u}_{z}\right| .
\end{aligned}
$$

From (i) it can be deduced that $\left\{\left|u_{\beta}\right|_{X}\right\}_{\beta>0}$ is bounded. Hence there exists a subsequence of $\left\{u_{\beta}\right\}_{\beta>0}$, denoted by the same symbol and $v \in X$ such that $u_{\beta} \rightarrow v$ strongly in $L^{p}(\Omega), p<\frac{n}{n-1}$, and weakly in $L^{2}(\Omega)$ as $\beta \rightarrow 0$. By Proposition 1.1 and (i)

$$
\begin{aligned}
& \frac{1}{2}|v|_{L^{2}}^{2}+\int_{\Omega}|\nabla v| \leq \frac{1}{2} \underline{\lim }\left|u_{\beta}\right|_{L^{2}}^{2}+\underline{\lim } \int_{\Omega}\left|\nabla u_{\beta}\right| \\
& \quad \leq \overline{\lim }\left(\frac{1}{2}\left|u_{\beta}\right|_{L^{2}}^{2}+\int_{\Omega}\left|\nabla u_{\beta}\right|\right) \leq \frac{1}{2}\left|\hat{u}_{z}\right|_{L^{2}}^{2}+\int_{\Omega}\left|\nabla \hat{u}_{z}\right| .
\end{aligned}
$$

Taking the limit with respect to $\beta \rightarrow 0^{+}$in (4.1) we obtain

$$
|T v-z|_{Y} \leq\left|T \hat{u}_{z}-z\right|_{Y}
$$

Combining (4.2) and (4.3) we find that $v$ is a minimal solution to $\left(\mathrm{P}_{0}\right)$. But the minimal solution to $\left(\mathrm{P}_{0}\right)$ is necessarily unique and therefore $\hat{u}_{z}=v$. Since $T u_{\beta} \rightarrow T \hat{u}_{z}$ weakly in $Y$ and $\left|T u_{\beta}-z\right| \rightarrow\left|T \hat{u}_{z}-z\right|$ it follows that $T u_{\beta} \rightarrow T \hat{u}_{z}$ strongly in $Y$. If $z \in$ range $(T \mid K)$ then $T \hat{u}_{z}=z$ and (iv) follows from (4.1) and (4.2).

We consider now the case where noise is admitted in the data. To obtain convergence and convergence rate of $u_{\beta}$ to $\hat{u}_{z}$, additional assumptions will be used. We consider

$$
\min \frac{1}{2}\left|T u-z_{\delta}\right|^{2}+\frac{\beta}{2}|u|_{L^{2}}^{2}+\beta \int_{\Omega}|\nabla u| \text { over } u \in K \cap X
$$


where

$$
\left|z-z_{\delta}\right|_{Y} \leq \delta
$$

The solution to $\left(\mathrm{P}_{\beta, \delta}\right)$ will again be denoted by $u_{\beta}$, i.e. we omit the dependence on $\delta$.

To interpret properly the assumptions that will be used below we reconsider the optimality condition for the minimal solution $\hat{u}_{z}$. For a convex set $C$ in $X$ the tangent cone to $C$ at $u \in C$ is defined by

$$
T(C, u)=\left\{v \in X: \exists v_{n} \in C, \lambda_{n}>0 \text { with } \lim _{n \rightarrow \infty} \lambda_{n}\left(v_{n}-u\right)=v\right\},
$$

the limit being taken in $X$, and the negative polar cone $N(C, u) \subset X^{*}$ at $u \in C$ is given by

$$
N(C, u)=T(C, u)^{-}=\left\{v \in X^{*}:\langle v, \bar{u}\rangle_{X^{*}, X} \leq 0 \text { for all } \bar{u} \in T(C, u)\right\} .
$$

Note that $\hat{u}_{z}-\operatorname{div} \Lambda_{z}$ defines an element of $X^{*}$ which we denote by the same symbol. The action of $\hat{u}_{z}-\operatorname{div} \Lambda_{z}$ on $\varphi \in X=L^{2}(\Omega) \times B V(\Omega)$ is given by

$$
\left\langle\hat{u}_{z}-\operatorname{div} \Lambda_{z}, \varphi\right\rangle_{X^{*}, X}=\left(\hat{u}_{z}, \varphi\right)_{L^{2}}+\left\langle\Lambda_{z}, \nabla \varphi\right\rangle_{M^{n * *}, M^{n}}
$$

Referring to Proposition 4.1 we have

$$
\operatorname{div} \Lambda_{z}-\hat{u}_{z} \in T\left(\mathcal{S}, \hat{u}_{z}\right)^{-} .
$$

We shall denote the intersections of $\operatorname{ker} T$ and $K$ with $B V$ by $\operatorname{ker}_{B V} T$ and $K_{B V}$ respectively. It is simple to argue that

$$
T\left(\mathcal{S}, \hat{u}_{z}\right)^{-} \subset T\left(K_{B V}, \hat{u}_{z}\right) \cap \operatorname{ker}_{B V} T .
$$

Definition 4.3. The minimal solution $\hat{u}_{z}$ is called qualified if

$$
T\left(\mathcal{S}, \hat{u}_{z}\right)=T\left(K_{B V}, \hat{u}_{z}\right) \cap \operatorname{ker}_{B V} T .
$$

Clearly $\hat{u}_{z}$ is qualified if $\operatorname{ker}_{B V} T=\{0\}$ or if $K_{B V}=B V(\Omega)$. If

$$
0 \in \operatorname{int}\left(K_{B V}-\operatorname{ker}_{B V} T\right)
$$

then $\hat{u}_{z}$ is qualified [1]. If

$$
K_{B V}=\left\{u \in X: f_{i}(u) \leq b_{i}, i=1, \ldots, M\right\},
$$

with $M$ fixed, $f_{i} \in X^{*}, b_{i} \in \mathbb{R}$, then one can proceed as in ([4], Prop. 3) to show that every element of $K_{B V}$ is qualified.

Proposition 4.4. If $\hat{u}_{z}$ is qualified then

$$
\operatorname{div} \Lambda_{z}-\hat{u}_{z} \in \overline{T\left(K_{B V}, \hat{u}_{z}\right)^{-}+\left(\operatorname{ker}_{B V} T\right)^{-}}{ }^{*},
$$

where the bar with index $X^{*}$ denotes closure in $X^{*}$.

Proof. Due to (4.5) and the qualification condition

$\operatorname{div} \Lambda_{z}-\hat{u}_{z} \in\left(T\left(K_{B V}, \hat{u}_{z}\right) \cap \operatorname{ker}_{B V} T\right)^{-}={\overline{T\left(K_{B V}, \hat{u}_{z}\right)^{-}+\left(\operatorname{ker}_{B V} T\right)^{-}}}^{X^{*}}$, the last identity being well-known for polar cones ([1], p. 175). 
Let us note that $\left(\operatorname{ker}_{B V} T\right)^{-}$cannot be replaced by $\overline{R g\left(T^{\prime}\right)}$ since $X$ is not reflexive and hence $\overline{R g\left(T^{\prime}\right)}$ may be a strict subset of $\left(\operatorname{ker}_{B V} T\right)^{-}$. Here $T^{\prime}: Y^{*} \rightarrow X^{*}$ denotes the conjugate of $T \mid X \in \mathcal{L}(X, Y)$. Let us also note that due to the assumption that $T \in \mathcal{L}\left(L^{2}(\Omega), Y\right)$ the elements in the range of $T^{\prime}$ can be identified with elements of $L^{2}$. The condition, which insures strong convergence of $u_{\beta}$ to $\hat{u}_{z}$ is given by (H1)

$$
-\hat{u}_{z} \in \overline{-\operatorname{div} \Lambda_{z}+T\left(K_{B V}, \hat{u}_{z}\right)^{-}+R g T^{*}} L_{2} .
$$

Here the bar with index $L_{2}$ denotes the closure in $L^{2}$. Since $R g T^{*} \subset$ $L^{2}(\Omega)$, it is assumed implicitly in (H1) that $T\left(K_{B V}, \hat{u}_{z}\right)^{-}-\operatorname{div} \Lambda_{z}$ can be identified with a subset of $L^{2}(\Omega)$. If $T\left(K_{B V}, \hat{u}_{z}\right)^{-}$equals $\{0\}$, it is thus required that $\operatorname{div} \Lambda_{z}$ can be identified with an element of $L^{2}(\Omega)$. We shall discuss this requirement after Theorem 4.6. Under the additional assumption that

$$
-\hat{u}_{z} \in-\operatorname{div} \Lambda_{z}+T\left(K_{B V}, \hat{u}_{z}\right)^{-}+R g T^{*}
$$

rate of convergence can be proved.

Theorem 4.5 .

i) If $(H 1)$ holds and $\beta \rightarrow 0^{+}, \delta^{2} / \beta(\delta) \rightarrow 0$ then the solutions $u_{\beta}$ to $\left(P_{\beta, \delta}\right)$ converge strongly to $\hat{u}_{z}$ in $L^{2}(\Omega)$.

ii) If (H2) holds and $\beta \rightarrow 0^{+}, \beta \sim \delta$, then

$$
\left|u_{\beta}-\hat{u}_{z}\right|_{L^{2}}=O(\sqrt{\beta})
$$

and

$$
\left|T u_{\beta}-\hat{z}\right|_{Y}=O(\beta)
$$

Proof. By the definition of $u_{\beta}$

$$
\begin{aligned}
& \frac{1}{2}\left|T u_{\beta}-z_{\delta}\right|_{Y}^{2}+\frac{\beta}{2}\left|u_{\beta}\right|_{L^{2}}^{2}+\beta \int_{\Omega}\left|\nabla u_{\beta}\right| \\
\leq & \frac{1}{2}\left|T \hat{u}_{z}-z_{\delta}\right|_{Y}^{2}+\frac{\beta}{2}\left|\hat{u}_{z}\right|_{L^{2}}^{2}+\beta \int_{\Omega}\left|\nabla \hat{u}_{z}\right|,
\end{aligned}
$$

and hence

$$
\begin{aligned}
& \frac{1}{2}\left|T u_{\beta}-\hat{z}\right|_{Y}^{2}+\frac{\beta}{2}\left|u_{\beta}-\hat{u}_{z}\right|^{2} \\
& \quad \leq \frac{1}{2}\left|T \hat{u}_{z}-z_{\delta}\right|^{2}+\frac{1}{2}\left|T u_{\beta}-\hat{z}\right|^{2}-\frac{1}{2}\left|T u_{\beta}-z_{\delta}\right|^{2} \\
& \quad+\frac{\beta}{2}\left|\hat{u}_{z}\right|^{2}+\frac{\beta}{2}\left|u_{\beta}-\hat{u}_{z}\right|^{2}-\frac{\beta}{2}\left|u_{\beta}\right|^{2}+\beta\left(\int_{\Omega}\left|\nabla \hat{u}_{z}\right|-\int_{\Omega}\left|\nabla u_{\beta}\right|\right) .
\end{aligned}
$$

Esaim: Cocv, December 1997, Vol. 2, Pp. 359-376 
Using $a^{2}+b^{2}-|a+b|^{2}=-2(a, b)$ twice yields

$$
\begin{aligned}
& \frac{1}{2}\left|T u_{\beta}-\hat{z}\right|_{Y}^{2}+\frac{\beta}{2}\left|u_{\beta}-\hat{u}_{z}\right|^{2} \\
& \leq\left(T u_{\beta}-\hat{z}, z_{\delta}-\hat{z}\right)_{Y}+\beta\left(\hat{u}_{z}, \hat{u}_{z}-u_{\beta}\right)+\beta\left(\int_{\Omega}\left|\nabla \hat{u}_{z}\right|-\int_{\Omega}\left|\nabla u_{\beta}\right|\right) \\
& \leq\left(T u_{\beta}-\hat{z}, z_{\delta}-z\right)+\left(T u_{\beta}-\hat{z}, z-\hat{z}\right)+\beta\left(\hat{u}_{z}, \hat{u}_{z}-u_{\beta}\right) \\
& \quad+\beta\left(\int_{\Omega}\left|\nabla \hat{u}_{z}\right|-\int_{\Omega}\left|\nabla u_{\beta}\right|\right) \\
& \leq\left(T u_{\beta}-\hat{z}, z_{\delta}-z\right)+\beta\left(\hat{u}_{z}, \hat{u}_{z}-u_{\beta}\right)+\beta\left(\int_{\Omega}\left|\nabla \hat{u}_{z}\right|-\int_{\Omega}\left|\nabla u_{\beta}\right|\right) .
\end{aligned}
$$

Let $\varepsilon>0$ be arbitrary and by (H1) choose $w \in Y, \eta \in T\left(K_{B V}, \hat{u}_{z}\right)^{-}$, $\mu \in L^{2}(\Omega)$ such that

$$
\hat{u}_{z}=\operatorname{div} \Lambda_{z}-\eta+T^{*} w+\mu, \quad|\mu|_{L^{2}} \leq \varepsilon .
$$

The $L^{2}$-inner product $\left(\hat{u}_{z}, \hat{u}_{z}-u_{\beta}\right)$ can be interpreted as duality pairing $\left(\hat{u}_{z}, \hat{u}_{z}-u_{\beta}\right)_{X^{*}, X}$ and hence

$$
\begin{aligned}
\frac{1}{2} \mid & T u_{\beta}-\left.\hat{z}\right|_{Y} ^{2}+\frac{\beta}{2}\left|u_{\beta}-\hat{u}_{z}\right|_{L^{2}}^{2} \\
& \leq\left(T u_{\beta}-\hat{z}, z_{\delta}-z\right)_{Y}+\beta\left(T^{*} w, \hat{u}_{z}-u_{\beta}\right)_{X^{*}, X} \\
& +\beta\left(\Lambda_{z}, \nabla u_{\beta}-\nabla \hat{u}_{z}\right)_{M^{n, *}, M^{n}}+\beta\left(\mu, \hat{u}_{z}-u_{\beta}\right)_{L^{2}} \\
& +\beta\left(\eta, u_{\beta}-\hat{u}_{z}\right)_{X^{*}, X}+\beta\left(\int_{\Omega}\left|\nabla \hat{u}_{z}\right|-\int_{\Omega}\left|\nabla u_{\beta}\right|\right) \\
& \leq\left(\delta+\beta|w|_{Y}\right)\left|T u_{\beta}-\hat{z}\right|+\beta \varepsilon\left|u_{\beta}-\hat{u}_{z}\right|_{L^{2}} .
\end{aligned}
$$

The following argument is now standard [4]. The last inequality can be expressed as

$$
a^{2}+b^{2} \leq 2 A a+2 B b
$$

where $a=\left|T u_{\beta}-\hat{z}\right|, b=\sqrt{\beta}\left|u_{\beta}-\hat{u}_{z}\right|, A=\left(\delta+\beta|w|_{Y}\right)$ and $B=\sqrt{\beta} \varepsilon$.

It is simple to argue that (4.9) implies

$$
a \leq 2 A+B \text { and } b \leq A+2 B,
$$

and (i) follows. If (H2) holds then one can take $\mu=0$ and (4.8) implies

$$
\left|T u_{\beta}-\hat{z}\right|_{Y}^{2}+\beta\left|u_{\beta}-\hat{u}_{z}\right|^{2} \leq 2\left(\delta+\beta|w|_{Y}\right)\left|T u_{\beta}-\hat{z}\right| .
$$

If $\delta \sim \beta$ then the asymptotic behavior asserted in (ii) follows.

Since the regularization term involves an $L^{2}$ as well as a $B V$-part we aim in the final result of this paper to improve the convergence properties of Theorem 4.5. We cannot expect convergence of $\int_{\Omega}\left|\nabla u_{\beta}-\nabla \hat{u}_{z}\right|$. In fact, let us assume that $\Omega=(0,1)$, and let $u_{\beta}$ be Heaviside functions with jump at $\frac{1}{2}+\beta, \beta \in\left(0, \frac{1}{2}\right)$. Then $u_{\beta} \in B V(0,1)$ and $\left|u_{\beta}-\bar{u}\right|_{L^{2}} \rightarrow 0, \quad\left|\int_{\Omega}\right| \nabla u_{\beta} \mid-$ $\int_{\Omega}|\nabla \bar{u}| \mid \rightarrow 0$ where $\bar{u}$ is the Heaviside function with jump at $\frac{1}{2}$. On the other hand $\int\left|\nabla u_{\beta}-\nabla \bar{u}\right|=2$ for all $\beta \in\left(0, \frac{1}{2}\right)$. 
THEOREM 4.6. In addition to the assumptions of Theorem 4.5 (ii) assume that div $\Lambda_{z} \in X^{*}$ can be identified with an element of $L^{2}(\Omega)$. Then

$$
\left|\int_{\Omega}\right| \nabla u_{\beta}\left|-\int_{\Omega}\right| \nabla \hat{u}_{z}|| \leq K\left|u_{\beta}-u_{z}\right|_{L^{2}}
$$

for a constant $K$ independent of $\beta \in(0,1]$. In particular

$$
\left|\int_{\Omega}\right| \nabla u_{\beta}\left|-\int_{\Omega}\right| \nabla \hat{u}_{z}||=O(\sqrt{\beta}) \text {. }
$$

Proof. By Proposition 4.1 we have

$$
\int_{\Omega}\left|\nabla \hat{u}_{z}\right|-\int_{\Omega}\left|\nabla u_{\beta}\right| \leq\left(\operatorname{div} \Lambda_{z}, u_{\beta}-\hat{u}_{z}\right) \leq\left|\operatorname{div} \Lambda_{z}\right|_{L^{2}}\left|u_{\beta}-\hat{u}_{z}\right|
$$

and further by (2.2) it follows that

$$
\begin{aligned}
\int_{\Omega} \mid & \nabla u_{\beta}\left|-\int_{\Omega}\right| \nabla \hat{u}_{z} \mid \leq \frac{1}{\beta}\left(\left(T^{*} T+\beta\right) u_{\beta}, \hat{u}_{z}-u_{\beta}\right)-\frac{1}{\beta}\left(T^{*} z_{\delta}, \hat{u}_{z}-u_{\beta}\right) \\
& =\left(u_{\beta}, \hat{u}_{z}-u_{\beta}\right)+\frac{1}{\beta}\left(T^{*}\left(T u_{\beta}-z_{\delta}\right), \hat{u}_{z}-u_{\beta}\right) \\
& \leq\left|u_{\beta}\right|_{L^{2}}\left|\hat{u}_{z}-u_{\beta}\right|_{L^{2}}+\frac{1}{\beta}\left(T^{*}\left(T u_{\beta}-\hat{z}+(\hat{z}-z)+z-z_{\delta}\right), \hat{u}_{z}-u_{\beta}\right) \\
& \leq\left|u_{\beta}\right|_{L^{2}}\left|\hat{u}_{z}-u_{\beta}\right|_{L^{2}}+\frac{1}{\beta}|T|_{\mathcal{L}\left(L^{2}, Y\right)}\left(\left|T u_{\beta}-\hat{z}\right|+\mid z-z_{\delta}\right)\left|\hat{u}_{z}-u_{\beta}\right| \\
& =C\left|\hat{u}_{z}-u_{\beta}\right|_{L^{2}}
\end{aligned}
$$

for some constant $C$ independent of $\beta>0$. Combined with (4.10) this gives the desired result.

REMARK 4.7. We consider several examples illustrating the condition $\operatorname{div} \Lambda_{z} \in L^{2}(\Omega)$, where $\Lambda_{z}$ satisfies the second condition stated in Proposition 4.1, i.e.

$$
\left\langle\Lambda_{z}, \mu-\nabla \hat{u}_{z}\right\rangle_{M^{n, *}, M^{n}}+\int_{\Omega}\left|\nabla \hat{u}_{z}\right| \leq \int_{\Omega}|\mu| \text { for all } \mu \in M^{n}(\Omega)
$$

which can equivalently be expressed as

$$
\left\langle\Lambda_{z}, \nabla \hat{u}_{z}\right\rangle_{M^{n, *}, M^{n}}=\int_{\Omega}\left|\nabla \hat{u}_{z}\right|
$$

and

$$
\left\langle\Lambda_{z}, \mu\right\rangle_{M^{n, *}, M^{n}} \leq \int_{\Omega}|\mu| \text { for all } \mu \in M^{n}(\Omega) .
$$

While these examples will be one-dimensional with $\Omega=(0,1)$, higher dimensional examples can be obtained by extending the functions by constant values in the remaining directions.

Let us briefly discuss the requirements that are imposed on $\Lambda_{z}$ by (4.11), (4.12) and $\Lambda_{z}$, div $\Lambda_{z} \in L^{2}(\Omega)$ in the one-dimensional case. First of all this necessitates that $\Lambda_{z} \in H^{1}(\Omega) \subset C(\Omega)$. Satisfying (4.11) amounts, formally, to setting $\Lambda_{z}=\operatorname{sign} \hat{u}_{z}^{\prime}$ at points where $\hat{u}_{z}$ is smooth but nonconstant. Since $\Lambda_{z} \in C(\Omega)$, condition (4.12) necessitates by the definition of $M(\Omega)$ as the dual of $C(\Omega)$ to choose $\Lambda_{z} \in C(\Omega)$ such that $\left|\Lambda_{z}(x)\right| \leq 1$ for all $x \in \Omega$. Esaim: CoCv, December 1997, Vol. 2, Pp. 359-376 
Summarizing we see that (at least for piecewise smooth $\hat{u}_{z}$ ) the existence of $\Lambda_{z}$ satisfying the specified requirements depends on the possibility of extending the graph of $\left\{\left(x, \operatorname{sgn} \hat{u}_{z}^{\prime}(x)\right): x\right.$ such that $\left.\hat{u}_{z}^{\prime}(x) \neq 0\right\}$ to a graph with domain $\Omega$ and $\left|\hat{u}_{z}^{\prime}(x)\right| \leq 1$ for all $x \in \Omega$. We now give three examples which illustrate the existence of $\Lambda_{z}$ - or the lack thereof.

(i) Let

$$
\hat{u}_{z}=\left\{\begin{array}{rll}
3 & \text { on } & (0,1 / 4) \\
4 & \text { on } & (1 / 4,1 / 2) \\
2 & \text { on } & (1 / 2,3 / 4) \\
1-x & \text { on } & (3 / 4,1),
\end{array}\right.
$$

then $\Lambda_{z}$ can be chosen as a $C^{\infty}(\Omega)$-function with $\left|\Lambda_{z}\right|_{C} \leq 1$ (so that (4.12) is satisfied) and such that it equals

- 1 in a neighborhood of $1 / 4$

- 1 in a neighborhood of $1 / 2$

- -1 in a neighborhood of $3 / 4$

- 1 on $(3 / 4,1)$.

Then $\Lambda_{z}$ thus defined satisfies (4.11), (4.12) and $\operatorname{div} \Lambda_{z} \in L^{2}(\Omega)$.

(ii) If $\hat{u}_{z}$ is chosen as in (i) but with $\hat{u}_{z}=x-1$ on $(3 / 4,1)$ instead of $1-x$, then satisfying (4.11) requires that $\Lambda_{z}$ equals

- 1 in a neighborhood of $1 / 4$

- 1 in a neighborhood of $1 / 2$

- 1 in a neighborhood of $3 / 4$

- 1 on $(3 / 4,1)$,

which can clearly not be satisfied by any function in $C^{\infty}(\Omega)$. So in this case there exists no regular $\Lambda_{z}$ satisfying (4.11) and (4.12).

(iii) For $\hat{u}_{z}$ given by

$$
\hat{u}_{z}=\left\{\begin{array}{rll}
\sin \pi x \alpha & \text { on } & \left(0, \frac{1}{2 \alpha}\right) \\
1 & \text { on } & {\left[\frac{1}{2 \alpha}, 1-\frac{1}{2 \alpha}\right]} \\
\sin \pi(\alpha(x-1)+1) & \text { on } & \left(1-\frac{1}{2 \alpha}, 1\right),
\end{array}\right.
$$

with $\alpha \in(1, \infty)$ one chooses $\Lambda_{z} \in C^{\infty}(\Omega),\left|\Lambda_{z}\right|_{C} \leq 1$ such that

$$
\Lambda_{z}=\left\{\begin{array}{rll}
1 & \text { on } & \left(0, \frac{1}{2 \alpha}\right) \\
-1 & \text { on } & \left(1-\frac{1}{2 \alpha}, 1\right) .
\end{array}\right.
$$

Clearly $\Lambda_{z}$ satisfies (4.11), (4.12) and $\operatorname{div} \Lambda_{z} \in L^{2}(\Omega)$. For $\alpha=1$ no such $\Lambda_{z}$ can be found.

\section{REFERENCES}

[1] J.-P. Aubin, I. Ekeland: Applied Nonlinear Analysis, Wiley-Interscience, New York, 1984.

[2] J. Baumeister: Stable Solutions of Inverse Problems, Vieweg, Braunschweig, 1987.

[3] E. Casas, K. Kunisch, , C. Pola: Regularization by functions of bounded variation and applications to image enhancement, preprint.

[4] G. Chavent, K. Kunisch: Convergence of Tikhonov regularization for constrained ill-posed inverse problems, Inverse Problems, 10 (1994), 63-76. Boston, 1985.

[5] V. Girault, P. A. Raviart: Finite Elements, Methods for Navier-Stokes Equations, Springer, Berlin, 1984.

[6] E. Giusti: Minimal Surfaces and Functions of Bounded Variation, Birkhäuser, Boston, 1984. 
[7] C. W. Groetsch: The Theory of Tikhonov Regularization for Fredholm Equations of the First Kind, Pitman, Boston, 1984.

[8] A. K. Louis: Inverse und schlechtgestellte Probleme, Teubner, Stuttgart, 1989.

[9] V.G. Mazja: Sobolev Spaces, Springer, Berlin, 1985.

[10] W. Rudin: Real and Complex Analysis, McGraw Hill, London, 1970.

[11] L. Rudin, S. Osher, E. Fatemi: Nonlinear total variation based noise removal algorithm, Physica D, 60 (1992), 259-268.

[12] R. Temam: Mathematical Problems in Plasticity, Gauthier-Villars, Kent, 1985.

[13] C. Vogel, M. Oman: Iterative methods for total variation denoising, preprint. 